This is the post-print (ie final draft post-refereeing) version of the following contribution published in the ZeFKo:

Evelyne Schmid, "Möglichkeiten und Grenzen des Völkerstrafrechts als Instrument gegen die illegale Ausbeutung von natürlichen Ressourcen und Landraub" in ZeFKo Zeitschrift für Friedens- und Konfliktforschung, (2017) Heft 1, 129-148. DOI: 10.5771/2192-1741-2017-1$\underline{129}$

\title{
Möglichkeiten und Grenzen des Völkerstrafrechts als Instrument gegen die illegale Ausbeutung von natürlichen Ressourcen und Landraub
}

\author{
Evelyne Schmid
}

Im September 2016 verkündete die Chefanklägerin des Internationalen Strafgerichtshofes (IStGH) in Den Haag in einem Strategiepapier, dass sich ihr Büro in Zukunft vermehrt mit Verbrechen befassen wolle, welche unter anderem Umweltzerstörung, illegale Ressourcenausbeutung oder Landnahme beinhalten oder zur Folge haben. Die Reaktionen auf diese Mitteilung reichten von Euphorie bis hin zu Vorwürfen, dass die Chefanklägerin damit dem Gerichtshof einen neuen Vorwand gäbe, sich ausschließlich mit dem Globalen Süden zu befassen. Was ist von dieser Kontroverse zu halten und welche Auswirkungen könnte die Ankündigung der Anklagebehörde auf die breiteren Bemühungen in der Vergangenheitsarbeit nach bewaffneten Konflikten und anderen Gewaltsituationen haben? Der vorliegende Beitrag bespricht das Strategiepapier der Chefanklägerin vor dem Hintergrund neuer Forschungsergebnisse, welche aufzeigen, dass Verletzungen von wirtschaftlichen, sozialen und kulturellen Menschenrechten mit völkerstrafrechtlichen Tatbeständen überlappen können.

Schlagworte: Völkerrecht, Völkerstrafrecht, Transitional Justice, Vergangenheitsarbeit, Menschenrechte, Internationaler Strafgerichtshof

\section{Einleitung}

Fünfzehn Jahre nach Einrichtung des Internationalen Strafgerichtshofes (IStGH), nach nunmehr über fünfzig Wahrheitskommissionen (Hayner 2011) und unzähligen Initiativen zum Umgang mit vergangenem Unrecht nach bewaffneten Konflikten oder anderen Gewaltsituationen erscheint es uns nicht weiter ungewöhnlich, dass Gesellschaften versuchen, Verletzungen gewisser sogenannter ziviler und bürgerlicher Menschenrechte zu ahnden. Zu solchen Verletzungen gehören zum Beispiel willkürliche Inhaftierungen, das Verschwindenlassen von Personen oder außergerichtliche Erschießungen. ${ }^{1}$

\footnotetext{
${ }^{1}$ Der vorliegende Beitrag greift Elemente der am 3. März 2016 in Bonn an der 48. Jahrestagung der Arbeitsgemeinschaft für Friedens- und Konfliktforschung gehaltenen Dankesrede zur Verleihung des Christiane Rajewsky-Preises auf und ergänzt diese mit der aktuellen Debatte zum Strategiepapier der Chefanklägerin des
} 
Demgegenüber ist die gezielte Aufarbeitung von sozioökonomischen und kulturellen Dimensionen des vergangenen Unrechts weitaus seltener Teil der im englischen Sprachraum inzwischen als Transitional Justice ${ }^{2}$ bezeichneten "Vergangenheitsarbeit« (Arbour 2007; Miller 2008; Schmid/Nolan 2014; Sharp 2012). Die Vernichtung oder Erschwerung von Lebensgrundlagen einer betroffenen Bevölkerung durch Umweltschäden oder Landraub und die damit einhergehenden Zwangsvertreibungen, Plünderungen oder Eigentumszerstörungen können Verletzungen wirtschaftlicher, sozialer und kultureller Menschenrechte (WSK-Rechte) darstellen. Zu den WSKRechten gehören beispielsweise das Recht auf Nahrung, Behausung, soziale Sicherheit, Arbeit, Gesundheit, Bildung oder das Recht zur Teilnahme am kulturellen Leben (für eine hervorragende, deutschsprachige Übersicht, siehe Kälin/Künzli 2013: insb. 122-128). Die Unterzeichnerstaaten der wichtigsten völkerrechtlichen Menschenrechtsabkommen haben sich verpflichtet, die Menschenrechte zu achten, zu schützen und zu erfüllen; d.h. die Staaten dürfen den Einzelnen nicht an der Ausübung seiner Rechte hindern (z.B. Menschen daran hindern, sich selber mit Nahrung zu versorgen); die Staaten müssen Einzelne vor Eingriffen Dritter schützen (z.B. Schutz vor gesundheitsgefährdender Umweltverschmutzung durch private Betriebe); und sie haben sich außerdem verpflichtet, die volle Verwirklichung der Rechte im Rahmen des innen Zumutbaren zu ermöglichen (z.B. adäquate Ausbildung von Lehrpersonal).

Dass Transitional Justice auf (einige) zivile und bürgerliche Rechte fokussiert und damit verbunden die WSK-Rechte vernachlässigt, ist nicht zwingend. Dies ist das Hauptergebnis meiner Forschungsarbeit, die im letzten Frühjahr gemeinsam mit einer Arbeit von Dorte Hühnert durch die Arbeitsgemeinschaft für Friedens- und Konfliktforschung mit dem Christiane Rajewsky-Preis ausgezeichnet wurde. ${ }^{3}$ Verliehen wurde mir der Preis für die Monographie Taking Economic, Social and Cultural Rights Seriously in International Criminal Law (Schmid 2015). Die Monographie befasst sich mit der Frage, ob WSK-Rechte in einer Art und Weise verletzt werden können, dass es juristisch zulässig ist, von der Erfüllung völkerstrafrechtlicher Tatbestände (wie z.B. Kriegsverbrechen oder Verbrechen gegen die Menschlichkeit) zu sprechen.

Im September 2016 veröffentlichte die Chefanklägerin des IStGH, Fatou Bensouda, ein Grundsatzpapier zu ihrer beabsichtigen Strategie bei der Auswahl von Fällen und der Prioritätensetzung. In diesem Strategiepapier kündigt die Chefanklägerin an, dass sich ihr Büro in der Zukunft vermehrt mit Verbrechen befassen will, welche unter anderem Umweltzerstörung, die illegale Ausbeutung von natürlichen Ressourcen oder Landnahme beinhalten oder darin resultieren. Diese Ankündigung wurde vielerorts positiv aufgenommen, löste aber auch einige negative Reaktionen aus.

In Anbetracht des neuen Strategiepapiers der Chefanklägerin zur Prioritätensetzung der Anklagebehörde am IStGH soll in diesem Beitrag dessen potenzielle Tragweite diskutiert werden.

IStGH. Ich danke der Jury der Arbeitsgemeinschaft für Friedens- und Konfliktforschung nochmals herzlich für die Anerkennung meiner Arbeit. Ich danke außerdem der Redaktion der ZeFKo für konstruktive Kommentare, sowie Rafael Federico Navarro Dalla Favera, BLaw, für die Durchsicht des Manuskriptes.

2 Der Uno-Generalsekretär definierte Transitional Justice als »the full range of processes and mechanisms associated with a society's attempt to come to terms with a legacy of large-scale past abuses«. (UN Secretary-General 2010: 2).

${ }^{3}$ Die Arbeitsgemeinschaft für Friedens- und Konfliktforschung zeichnete Dorte Hühnert für ihre Arbeit »New Kind of War - New Kind of Detention? How the Bush Administration Introduced the Unlawful Enemy Combatant« (Goethe-Universität Frankfurt/Main) aus. 
Darf und soll sich der IStGH mit Fakten befassen, welche Umweltzerstörung, illegale Ressourcenausbeutung oder Landraub und die damit einhergehenden sozioökonomischen Menschenrechtsverletzungen betreffen? Und welche Auswirkungen könnte eine solche strategische Neuausrichtung auf die breiteren Bemühungen im Rahmen der Vergangenheitsarbeit/Transitional Justice haben?

Vorneweg soll unbedingt festgehalten werden, dass der IStGH nicht die einzige völkerstrafrechtliche Institution darstellt, ${ }^{4}$ und auch keinesfalls als der wichtigste Akteur im Zusammenhang mit der Aufarbeitung einer gewaltsamen Vergangenheit eines Gemeinwesens gesehen werden darf. ${ }^{5}$ Dennoch bietet das Strategiepapier der Chefanklägerin einen Impuls, um zu diskutieren, inwieweit internationale Strafgerichte überhaupt die Möglichkeit haben, Menschenrechtsprobleme im Bereich der WSK-Rechte $\mathrm{zu}$ ahnden und welchen Beitrag völkerstrafrechtliche Aufmerksamkeit auf solche Rechtsverletzungen für die breiteren Debatten zur Aufarbeitung von gewaltsamen Situationen leisten könnte. Nach einem kurzen Überblick über die hier diskutierte Aussage im Strategiepapier der Anklagebehörde des IStGH und einigen Reaktionen darauf (Teil 2), erläutere ich in Teil 3 in groben Zügen meine Ergebnisse zur Reichweite des Völkerstrafrechts in wirtschaftlichen, sozialen und kulturellen Bereichen. Darauf aufbauend erkläre ich, warum Fatou Bensouda beigepflichtet werden kann, dass der IStGH die Möglichkeit hat, sich vermehrt Missbräuchen wie Landraub und der Zerstörung der natürlichen Lebensgrundlagen zuzuwenden. Im Teil 4 diskutiere ich die potenzielle Bedeutung dieser Aussage Fatou Bensoudas über den IStGH hinaus.

\section{Das Strategiepapier der Chefanklägerin}

Das (rechtlich nicht verbindliche) Strategiepapier bezweckt der Öffentlichkeit zu erklären, wie die Chefanklägerin und die von ihr geleitete Anklagebehörde des IStGH bei der Auswahl der Fälle für Untersuchungen und Gerichtsverfahren vorgehen. Es soll beschreiben, auf welche Aspekte die Chefanklägerin besonderen Wert legt. Insbesondere soll es in den Grundzügen transparent machen, wie Chefanklägerin Bensouda und ihr Personal die Prozesse zur Auswahl der Sachverhalte gestalten wollen, welche prima facie für die Eröffnung eines Verfahrens in Frage kommen und wie die Behörde bei der Prioritätensetzung vorzugehen gedenkt (Office of the Prosecutor of the International Criminal Court 2016a: 2, Ziff. 1).

\subsection{Die Ankündigung Fatou Bensoudas}

Im Wortlaut der Chefanklägerin: "[T]he Office will give particular consideration to prosecuting Rome Statute crimes that are committed by means of, or that result in, inter alia, the destruction of the environment, the illegal exploitation of natural resources or the illegal dispossession of land " (Office of the Prosecutor of the International Criminal Court 2016a: 14, Ziff. 41).

Die Auswahl der Sachverhalte und Fälle, die am IStGH weiterverfolgt werden sollen, ist eine der zentralsten und schwierigsten Entscheidungen, welche die Chefanklägerin in ihrer Funktion treffen muss. Mit dieser Auswahl beeinflusst sie in höchstem Maße das Profil des Gerichtshofes und

\footnotetext{
${ }_{4}^{4}$ Nationale Strafgerichte, Untersuchungs- oder Wahrheitskommissionen, hybride Gerichte und Ad-Hoc-Tribunale bleiben wichtige Akteure im Völkerstrafrecht und haben jeweils unterschiedliche Zuständigkeiten.

${ }^{5}$ Für die allermeisten Betroffenen von Gräueltaten bleibt der IStGH eine weitentfernte Institution, mit der sie nie in Kontakt treten. Dazu siehe z.B. Okafor/Ngwaba 2014.
} 
bindet die Ressourcen der Institution. Dadurch ist die Prioritätensetzung bei der Fallauswahl unzweifelhaft mit sehr viel Verantwortung verbunden. Das Strategiepapier der Chefanklägerin ist vor dem Hintergrund zu sehen, dass sich der IStGH in den bisherigen Verfahren exklusiv mit dem afrikanischen Kontinent befasst hat - was zumindest als Vorwand für die inzwischen erfolgten Austritte von Südafrika, Gambia und Burundi diente. ${ }^{6}$ Zudem hat sich der IStGH bisher "ausschliesslich « Fällen gewidmet, in denen Verbrechen in Situationen bewaffneter Konflikte begangen wurden (Vorwürfe zu Kriegsverbrechen oder Verbrechen gegen die Menschlichkeit und Genozid im Zusammenhang mit bewaffneten Konflikten), nicht aber solchen, die in völkerrechtlich als »Friedenszeiten « zu qualifizierenden Situationen auftreten, obwohl sie verschiedene Formen von Gewalt beinhalten können.

Gegenüber der Agence France Press erklärte Helen Brady, Mitarbeiterin der Anklagebehörde des IStGH, dass die vermehrte Aufmerksamkeit auf Umweltschäden, illegale Ressourcenausbeutung und Landnahme einschliesslich der Verfolgung von Verbrechen an Kindern, geschlechtsspezifischer Gewalt und kultureller Zerstörung dazu beitragen werde, "dass der Gerichtshof ein Profil für das 21. Jahrhundert und darüber hinaus erhalte (Agence France Press 2016). Aus dem Römischen Statut, der vertraglichen Grundlage des IStGH, ist für die Fallauswahl der Fokus auf das Kriterium der Schwere der Verbrechen (gravity) vorgegeben. ${ }^{7}$ Bisher fand am Gerichtshof wenig kritische Auseinandersetzung mit diesem sozial-konstruierten und alles andere als selbstredenden Kriterium statt (DeGuzman 2012: 284-289). Begrüßenswert ist deshalb Fatou Bensoudas implizite Aussage, dass das Kriterium der Schwere mutmaßlicher Verbrechen in höchstem Masse auslegungsbedürftig sei. Es kann tatsächlich nicht angenommen werden, dass die Schwere eines mutmaßlichen Verbrechens durch alle Akteure gleich beurteilt wird (Office of the Prosecutor of the International Criminal Court 2016a: 13, Ziff. 36). ${ }^{8}$ Während inr Vorgänger, Luis Moreno Ocampo, den völlig unrealistischen Standpunkt vertrat, dass er unparteiisch alle Sachverhalte untersuchen wolle, welche mutmaßlich die Tatbestandselemente eines völkerstrafrechtlichen Verbrechens erfüllen (ICC Observers 2009), ist die neue Vorsteherin der Anklagebehörde somit erfrischend ehrlich in der Aussage, dass sich der IStGH niemals der Gesamtheit aller völkerstrafrechtlichen Gräueltaten wird annehmen können.

\subsection{Reaktionen und Diskussion}

Die offizielle Reaktion der am IStGH beteiligten Staaten auf das Strategiepapier fiel positiv aus. Die jährliche Vertragsstaatenkonferenz (Assembly of States Parties, ASP) verabschiedete im

${ }^{6}$ Russland ist nicht aus dem System des Gerichtshofes ausgetreten, sondern hat angekündigt, die russische Unterschrift des Römischen Statuts zurückzuziehen. Russland hat das Statut jedoch nie ratifiziert und der Rückzug der Unterschrift hat juristisch deshalb wenig Bedeutung, ist aber ein politischer Rückschlag für den Gerichtshof. Der neu gewählte Präsident Gambias, Adama Barrow, kündigte am 2. Dezember 2016 an, dem IStGH wieder beitreten zu wollen.

${ }^{7}$ «Die schwersten Verbrechen, welche die internationale Gemeinschaft als Ganzes berühren». ${ }^{8}$ So schreibt sie im Grundsatzpapier, dass weit mehr Fälle zulässig wären als solche, welche die Anklagebehörde priorisieren kann/will: »However, given that many cases might potentially be admissible under article 17 [of the Rome Statute], the Office may apply a stricter test when assessing gravity for the purposes of case selection than that which is legally required for the admissibility test under article 17.« (Office of the Prosecutor of the International Criminal Court 2016a: Ziff. 36). 
November 2016 im Konsens eine Resolution, in der die Staaten das Strategiepapier willkommen heißen (ASP 2016: 42, Ziff. 47).

Die weiteren öffentlichen Reaktionen auf das Strategiepapier lassen sich grob in drei Gruppen einteilen, wobei die Übergänge zwischen den ersten beiden fließend sind. Bei der Ersten liegt der Fokus auf den mutmaßlichen positiven Auswirkungen des Strategiepapiers für die Umwelt. Eine zweite Gruppe von Reaktionen beinhaltet Rezensionen, welche die potenziellen Auswirkungen des Strategiepapiers auf menschenrechtliche Belange einschätzen und dabei entweder positiv oder neutral über das Strategiepapier berichten. Die dritte Gruppe umfasst skeptische Reaktionen auf die Aussage Fatou Bensoudas.

\subsubsection{Umweltzerstörung}

Einige Reaktionen konzentrierten sich auf die umweltbezogenen Auswirkungen der Ankündigung Fatou Bensoudas. Diese Auswirkungen werden in den bisherigen öffentlich auffindbaren Reaktionen positiv bis enthusiastisch eingeschätzt. So hofft Tara Smith (2016), dass eine strafrechtliche Verurteilung von Umweltsündern als Katalysator für die Industrie dienen könnte, schmutzige Geschäfte zu beenden. Franziska Kring (2016) zeigt sich gar optimistisch, dass der IStGH in Zukunft möglicherweise Sachverhalte abklären könnte, in denen Folgen des Klimawandels auf die menschliche Gesundheit im Fokus stehen. Unter den Reaktionen mit dem Schwerpunkt Umweltzerstörung findet sich auch mindestens eine Stimme, die sich für eine weniger anthropozentrische Sicht der internationalen Strafjustiz einsetzt und Schäden an Fauna und Flora als solche verfolgt sehen möchte (Seachange 2016). Öffentliche, negative Stellungnahmen zu den potenziellen Auswirkungen von Bensoudas Strategiepapier auf die Umwelt lassen sich zumindest bisher nicht finden.

\subsubsection{Menschenrechte}

Die hauptsächlich menschenrechtsbezogenen Stellungnahmen auf das Strategiepapier fielen ebenfalls mehrheitlich positiv aus. Insbesondere erhoffen sich einige nun baldige Fortschritte im Zusammenhang mit mutmaßlichen Verbrechen in Kambodscha. Ende 2014 überreichten eine NGO und eine Anwaltskanzlei der Anklagebehörde des IStGH eine umfangreiche Dokumentation, in der Mitgliedern der kambodschanischen Elite vorgeworfen wird, Landraub und Zwangsvertreibungen von bis zu 350.000 Personen vorgenommen zu haben. Die damit verbundenen Sachverhalte seien als Verbrechen gegen die Menschlichkeit zu qualifizieren und verdienten eine offizielle Untersuchung des IStGH (Global Diligence LLP 2014). Die Ankündigung der Chefanklägerin erweckt nun die Hoffnung, dass die Anklagebehörde demnächst eine Vorverfahrenskammer um Erlaubnis bitten könnte, eine offizielle Untersuchung dieser Vorwürfe einzuleiten, um damit konkrete Verfahren ins Rollen bringen zu können. Falls dies eintreffen sollte, würde sich der Gerichtshof erstmals mit Vorwürfen befassen, die hauptsächlich wirtschaftliche und soziale Menschenrechtsprobleme betreffen und welche außerhalb eines bewaffneten Konfliktes stattfanden bzw. stattfinden.

Die Washington Post wies richtigerweise darauf hin, dass die Ankündigung Bensoudas nicht dahingehend zu verstehen sei, dass die Chefanklägerin die Definition der völkerstrafrechtlichen Tatbestände unter dem Römischen Statut ändern wolle (was sie auch gar nicht kann). Sie zitierte Alex Whiting, Professor an der Harvard Law School, mit der korrekten Feststellung, dass die Chefanklägerin sich weiterhin auf die im Römischen Statut völkerrechtlich verankerten Verbrechen abstützen wird und das anwendbare Recht unverändert bleibt. Vielmehr gehe es Bensouda darum, vermehrte Aufmerksamkeit auf Verhaltensweisen zu lenken, welche die Tatbestandselemente völkerrechtlicher Verbrechen mutmaßlich erfüllen, aber bisher vom Strafgerichtshof kaum beachtet wurden (Taylor 2016). Dass mit der Aufmerksamkeit zugunsten von Umweltzerstörung, Landnahme oder Ressourcenausbeutung unter Umständen auch die Wahrscheinlichkeit steigt, 
dass künftig nebst Rebellen, Regierungs- oder Militärangehörigen auch Führungspersonal in Unternehmungen vom Radar des Büros der Chefanklägerin erfasst werden könnten, ist eine mögliche Nebenfolge. ${ }^{9}$ Das irische Zentrum für Menschenrechte der National University of Ireland in Galway entschied sich in diesem Zusammenhang nach der Ankündigung Bensoudas, seinen international renommierten Sommerkurs zum Völkerstrafrecht dieses Jahr dem Thema »Corporate Crimes « zu widmen. Dazu lud es unter anderem Richard J. Rogers, den federführenden Anwalt des oben genannten Antrags zu Kambodscha, ein (Irish Centre for Human Rights 2017).

\subsubsection{Negative Reaktionen}

Die Ankündigung Bensoudas löste auch skeptische Reaktionen aus. Christine Schwöbel-Patel (2016) äußerte sich etwa dahingehend, dass die Ankündigung nichts anderes als ein »Re-Brand « sei, welcher der Anklagebehörde erlaube, die Zuständigkeit des Gerichtshofes aufzublähen. Da Landraub schwergewichtig im Globalen Süden stattfindet, sei die Ankündigung der Chefanklägerin ein neuer Vorwand für den IStGH, sich weiterhin ausschließlich mit dem Süden zu befassen, ohne dabei die Komplizenschaft des Westens behandeln zu müssen. Damit werde die Wahrnehmung, der IStGH befasse sich nicht mit dem Westen, weiterhin Bestand haben (Schwöbel-Patel 2016). Ihre dezidierte Meinung hielt sie in einem Blogeintrag fest, welcher anschliessend unter anderem in einem Op-Ed auf der Titelseite der New York Times zitiert wurde (Cruvellier 2016; Karnavas 2016). Schwöbel-Patel kritisierte außerdem, dass die Anklagebehörde mit dem Strategiepapier einen »turn to explicit symbolism « vornehmen würde. Damit ist gemeint, dass der Gerichtshof seine Rolle zunehmend darin sehe, die sog. expressive Funktion der internationalen Strafjustiz zu maximieren, d.h. die Idee, dass ein Gerichtsprozess oder -urteil die Unhaltbarkeit gewisser Verhaltensweisen unterstreichen solle (dazu Kersten 2016).

Ohne explizite Bezugnahme zum Strategiepapier, aber in diesem Kontext als zur Vorsicht mahnende Äußerung zu verstehen, ist die Aussage eines anderen Bloggers, Patryk I. Labuda. Labuda ruft den IStGH dazu auf "zu seinem Hauptmandat zurückzukehren «, anstatt sein Mandat "uferlos « auszulegen (Labuda 2016). Teil 3 bespricht ausführlich, ob die Umsetzung der Ankündigung Bensoudas einer Abkehr vom Hauptmandat des Gerichtshofes gleichkommen würde.

Was ist von der Kritik Schwöbel-Patels zu halten? Da ihr Blogeintrag die bisher einzige auffindbare, argumentierte negative Reaktion auf das Strategiepapier darstellt, aber davon auszugehen ist, dass wohl auch andere Skepsis gegenüber dem Strategiepapier hegen, soll hier auf die im Blogeintrag geäußerten Punkte eingegangen werden. Zuerst befasse ich mich mitder Vermutung, die Ankündigung Fatou Bensoudas stelle bloß sicher, dass sich der Internationale Strafgerichtshof weiterhin ausschließlich mit dem Globalen Süden befassen könne (Schwöbel-Patel 2016). Es bestehen wenig Zweifel, dass völkerstrafrechtlich relevante Landnahme, illegale Ressourcenausbeutung und Umweltzerstörung vielfach im Süden erfolgen und dass die Ankündigung des IStGH deshalb potenziell Sachverhalte betreffen wird, bei denen zumindest die Endergebnisse außerhalb der Industrieländer stattfinden. Nichtsdestotrotz scheint die Skepsis gegenüber der Aussage Fatou Bensoudas übertrieben. Das Strategiepapier eignet sich meines Erachtens nicht, der Chefanklägerin eine Absicht zu unterstellen, »den Westen« ungeschoren davon kommen zu lassen. Erstens schließt die Ankündigung nicht aus, dass sich die Anklagebehörde nicht auch mit der Komplizenschaft westlicher Akteure zu befassen gedenkt, auch

\footnotetext{
${ }^{9}$ Firmen als juristische Person können nicht vor dem IStGH belangt werden, da Art. 25 Abs. 1 des Römischen Statuts vorschreibt, dass der Gerichtshof ausschließlich Gerichtsbarkeit über natürliche Personen hat. Führungskräfte in Unternehmungen, nicht aber die Unternehmen als solche, können somit als natürliche Personen vor dem IStGH belangt werden.
} 
wenn naturgemäß die juristischen Herausforderungen des Nachweises der strafrechtlichen Verantwortlichkeit von geografisch und institutionell von den Endresultaten der Missbräuche getrennten Akteuren groß sind. Zweitens wäre die möglicherweise bevorstehende Eröffnung einer formellen Untersuchung der Vorwürfe zu Kriegsverbrechen durch amerikanische Truppen in Afghanistan sowie zu den vermuteten Geheimgefängnissen in Europa ein Gegenindiz (zum vorläufigen Bericht der Chefanklägerin dazu, siehe Office of the Prosecutor of the International Criminal Court 2016b: 43-51, insb. Ziff. 198).

Was ist von den Bedenken Schwöbel-Patels zu halten, die Chefanklägerin wende sich dem Symbolismus zu? Ihre Aussage, dass die Anklagebehörde mit einem vermehrten Fokus auf Umweltschäden, Landnahme oder Ressourcenausbeutung nun einen »turn to explicit symbolism « vornehmen würde und die Aufmerksamkeit damit auf »nicht-physische Gewalt« lenken würde, ist nicht haltbar (Schwöbel-Patel 2016). Völkerstrafrechtlich relevante Verhaltensweisen im Zusammenhang, z.B., mit gewalttätigen Landnahmen im Rahmen einer zwangsweisen Überführung einer Bevölkerung oder unmenschliche Behandlungen und sklavereiähnliche Praktiken bei illegaler Ressourcenausbeutung können sehr wohl die physische Integrität der Opfer verletzen und beinhalten somit auch physische Gewalt (dazu auch Sharp 2013: 5). Zudem scheint deren Bekämpfung nicht inhärent mehr oder weniger symbolisch als die Bekämpfung anderer Verhaltensweisen. Dass sich die Chefanklägerin bewusst(er) mit der symbolischen Wirkung des Gerichtshofes auseinandersetzt, ist kein Makel, sondern kann durchaus positiv gewertet werden (Meijers/Glasius 2016: 444-445; Schmid 2015: 334-336).

Zusammenfassend liefert das Strategiepapier Anhaltspunkte, wie die Chefanklägerin vorgehen will, um »die schwersten Verbrechen, welche die internationale Gemeinschaft als Ganzes berühren « (Vereinte Nationen 1998: Präambel) zu identifizieren. Sie sagt dabei implizit, dass als Kriterium nicht »nur « die Anzahl der mit Feuerwaffen oder Machete oder anderen Formen direkter physischer Gewalt getöteter oder verletzter Personen maßgebend sei.

Auch wenn sie den Begriff "WSK-Rechte « nicht erwähnt, so können insbesondere die illegale Landnahme und der Hinweis auf den (sozio)ökonomischen Schaden von Verbrechen durchaus als Bekenntnis gewertet werden, dass auch WSK-Rechtsdimensionen völkerstrafrechtlicher Tatbestände gemeint sind, d.h. unter anderem Dimensionen von Verbrechen, welche die Zerstörung oder Erschwerung von Lebensgrundlagen der betroffenen Gemeinschaft tangieren. Unzweideutig weist die Aussage Bensoudas darauf hin, dass das Büro der Chefanklägerin davon ausgeht, der Gerichtshof habe bisher gewisse Aspekte von Verbrechen kaum bis gar nicht beachtet, obwohl die rechtlichen Grundlagen dazu bestehen würden.

Die Feststellung möglicher »Blindflecken« (Herik 2014) im Fokus der aktuellen Völkerstrafrechtspraxis deckt sich, wie oben erwähnt, mit den Ergebnissen meiner eigenen Forschung zur Frage der völkerstrafrechtlichen Möglichkeiten, Aspekte von Verletzungen von WSK-Rechten zu ahnden. In der Folge soll deshalb überblicksartig erläutert werden, in welchem Zusammenhang Verletzungen von WSK-Rechten mit dem Völkerstrafrecht stehen, um dann in Teil 4 darauf einzugehen, welche Bedeutung diese Forschungsergebnisse sowie die Ankündigung der Chefanklägerin Bensouda für die Vergangenheitsarbeit haben könnten.

\section{WSK-Rechte und völkerstrafrechtliche Verbrechen}

Ausgangspunkt der Analyse zu WSK-Rechten im Völkerstrafrecht ist die Ungleichheit in der Wahrnehmung und Behandlung von dem was (insbesondere im deutschsprachigen Raum) in Lehrbüchern immer noch oft als inhärent verschiedene Kategorien (oder noch verwirrender: 
Generationen) von Menschenrechten bezeichnet wird: den zivilen und bürgerlichen Menschenrechten auf der einen Seite und den WSK-Rechten auf der anderen.

Wie anfangs erwähnt, besteht die Tendenz, WSK-Rechte in der aktuellen völkerstrafrechtlichen Praxis und auch der Arbeit von anderen Transitional-Justice-Mechanismen wie z.B. Wahrheitskommissionen oder Entschädigungsprogrammen zu vernachlässigen (Office of the United Nations High Commissioner for Human Rights 2014: 6). Massive Verletzungen von Menschenrechten aber auch Massenverbrechen werden oft explizit mit Verletzungen von (einigen wenigen) zivilen und bürgerlichen Rechten gleichgesetzt, während z.B. Hunger, zerstörte und geplünderte Behausungen, verwehrte Bildungsmöglichkeiten oder miserable und diskriminierende Gesundheitsversorgung oft bloß als der diffuse und unvermeidliche Hintergrund wahrgenommen werden, vor dem sich die "schwersten" Verbrechen abspielen. Die Tendenz, WSKRechtsprobleme zu vernachlässigen, besteht häufig sogar dann, wenn z.B. Hinweise vorliegen, dass hinter der Zerstörung oder der Erschwerung von Lebensgrundlagen ein Kalkül liegt, etwa dass sich Menschen so aus einem bestimmten Gebiet vertreiben lassen (Andreu-Guzman 2012: 248).

Spätestens seit einer Rede der damaligen Uno-Menschenrechtshochkommissarin Louise Arbour (2007) ist der häufige Fokus auf (wenige) zivile und bürgerliche Rechte in den Bemühungen zur Vergangenheitsarbeit allerdings nicht mehr ohne Kritik. So weisen beispielsweise Stimmen aus Südafrika, Timor Leste, Peru, Kambodscha oder solche, die sich für Gender-Sensitivität in der Vergangenheitsarbeit aussprechen, darauf hin, dass in vielen Kontexten Verletzungen von WSKRechten ein prägendes Element des vergangenen Unrechts darstellten und dass dieses Unrecht ohne Rücksichtnahme auf sozioökonomische oder kulturelle Dimensionen nicht sinnvoll aufgearbeitet werden könne (für einen Überblick über die Debatte, z.B. Sharp 2012; Haldemann/Kouassi 2014; Cahill-Ripley 2014).

Die Literatur, welche sich zugunsten vermehrter Aufmerksamkeit gegenüber Aspekten von WSKMenschenrechtsproblemen in der Vergangenheitsarbeit ausspricht, nimmt über weite Strecken mehrheitlich an, dass die geltenden völkerrechtlichen Grundlagen wenig bis gar keinen Spielraum enthalten, den WSK-Rechten innerhalb des konzeptionellen Rahmens von Transitional Justice vermehrte Aufmerksamkeit zuteilwerden zu lassen. So müsse man zuerst den konzeptionellen Rahmen und die völkerrechtlichen Normen ausdehnen bzw. neue hinzufügen, bevor sich die Vergangenheitsarbeit vermehrt WSK-Rechten zuwenden könne (dazu ausführlich Schmid 2015: 22-40). In meiner Forschungsarbeit machte ich mir deshalb zur Aufgabe zu prüfen, ob die ungleiche Sicht auf die Menschenrechte in der Lehre und Praxis der Vergangenheitsarbeit tatsächlich im positiven Recht angelegt ist oder ob WSK-Rechte in Transitional-Justice-Prozessen aus anderen Gründen meist vernachlässigt werden.

Und zwar entschied ich mich, diese übergeordnete Frage im Völkerstrafrecht zu prüfen. Einerseits ist das Völkerstrafrecht der Bereich, in dem eine Befassung mit WSK-Rechten am schwierigsten erscheint. Damit verbunden schien mir zum anderen das Völkerstrafrecht derjenige Bereich zu sein, in dem ein überzeugender Nachweis der juristischen Relevanz von WSK-Rechten potenziell am meisten Auswirkungen über die Strafgerichte hinaus haben wird. Völkerstrafrechtlerlnnen stellen aus guten Gründen außerordentlich strenge Anforderungen an die juristische Auslegung. Nur das mit einem hohen Bestimmtheitsgrad umschriebene Verhalten darf gemäß den Gesetzmäßigkeitsgrundsätzen zu einer strafrechtlichen Verurteilung führen. Die veraltete aber immer noch verbreitete Sicht auf WSK-Rechte als juristisch schlecht fassbare Zielsetzungen führt dazu, dass Sachverhalte, die sozioökonomische Lebensbedingungen berühren, mitunter einen besonders schweren Stand bei Völkerstrafrechtlerlnnen haben. Wenn Juristen und Juristinnen an Gerichten, in Wahrheitskommissionen oder in der Wissenschaft davon ausgehen, dass WSK- 
Rechte inhärent vage sind und keine Aspekte beinhalten, welche unmittelbare und individuell einklagbare Verpflichtungen der Staaten setzen, werden sie die Überschneidung zwischen WSKRechtsverletzungen und völkerstrafrechtlichen Tatbeständen nicht für möglich halten. Wenn nun aber der Nachweis gelingen würde, dass sich das positive Völkerstrafrecht mit WSK-Rechten befassen kann und sich bereits befasst hat, dann sollte dies Auswirkungen auf die breitere Praxis in der Vergangenheitsarbeit haben, und zwar über die Strafgerichte hinaus. Daraus entstand die Forschungsfrage, ob es eine Überschneidung zwischen den anerkannten Völkerstrafrechtstatbeständen und WSK-Rechtsverletzungen gibt. Meine Hypothese war, dass es eine solche Überschneidung trotz anderslautenden Annahmen gibt.

Bei der Prüfung der Hypothese kamen unerwartet viele Beispiele zusammen, welche deutlich machen, dass das Völkerstrafrecht Verletzungen von WSK-Rechten in verschiedenen Tatbeständen miteinschließen kann. Zahlreiche internationale aber auch nationale und hybride Gerichtsverfahren oder Wahrheitskommissionen haben sich seit jeher mit Dimensionen von WSKRechten befasst, auch wenn sie dies nicht explizit so darstellen und auch wenn sich diese Überschneidung teilweise nur auf einzelne Aspekte eines Verfahrens beschränken. ${ }^{10}$ Dass WSKRechte - und damit verbunden auch Sachverhalte im Zusammenhang mit Umweltzerstörung, illegaler Ressourcenausbeutung oder Landnahme - durchaus einen Platz innerhalb des anerkannten Völkerstrafrechts haben, illustrieren die folgenden Beispiele.

Am 7. Juli 1946 verurteilte der oberste polnische Strafgerichtshof Arthur Greiser. Greiser war der Gauleiter des Reichsgau Warthelands, eines durch die Reichsregierung annektierten Gebiets, welches zusammen mit dem Reichsgau Danzig-Westpreußen und dem an die Provinz Schlesien angegliederten "Ostoberschlesien « und dem "Südostpreußen" die sogenannten eingegliederten Gebiete bildete. Greisers Aufgabe war - vereinfacht gesagt -, die Gegend so umzugestalten, dass Deutsche sich dort ansiedeln könnten und die polnischen Bewohnerlnnen diesen Plan nicht stören würden. Dass dies nicht geschehen konnte, ohne in gravierender Weise gegen die Menschenwürde der polnischen Menschen zu verstoßen, liegt auf der Hand. Aus Sicht des heutigen völkerrechtlichen Menschenrechtsschutzes lassen sich die Missbräuche als Verletzungen von verschiedenen WSK-Rechten qualifizieren, so z.B. dem Recht auf Bildung, Gesundheit, Arbeit oder der Teilnahme am kulturellen Leben. Greiser schuf insbesondere die juristischen Grundlagen für einen besonderen rechtlichen Status für die ethnischen Polen, das sog. Polenstatut, welches die betroffene Bevölkerung vollständig aus dem deutschen Recht herauslöste und in zahlreichen Bereichen gegenüber den Deutschen markant schlechterstellte: So verbot Greiser über dieses Rechtsregime der polnischen Bevölkerung, ihre Arbeitsstelle frei zu wählen, beutete ruchlos ihre Arbeitskraft aus oder verhinderte die Grundschulbildung der Kinder über ein tiefstes Minimalmass hinaus und verbot oder verunmöglichte die Teilnahme am kulturellen Leben (Prosecutor v. Greiser, Case no. 74, XIII Law Reports of Trials of War Criminals 70, 7. Juli 1946, Supreme National Tribunal: 73). Interessanterweise war Arthur Greiser die allererste Person, welche gestützt auf die Nürnberger Charta, die »Geburtsurkunde des modernen Völkerstrafrechts « (Werle 2016: Ziff. 15), verurteilt wurde. Der Strafprozess von Arthur Greiser ist aus sprachlichen und historiographischen Gründen nahezu vollends in Vergessenheit geraten, wurde aber in den letzten Jahren in der

${ }^{10}$ Dass kaum ein Strafgericht je von WSK-Rechten spricht, ist nicht erstaunlich. Das Mandat eines Strafgerichts ist die Bestimmung der Schuld oder Unschuld spezifischer Angeklagter und nicht die rechtliche Qualifikation des Sachverhaltes unter dem völkerrechtlichen Menschenrechtschutz. Zum Zusammenspiel des Völkerstrafrechts mit dem völkerrechtlichen Menschenrechtsschutz, siehe Schmid 2015: 67-73. 
Literatur aufgearbeitet (Epstein 2010) und für das Völkerstrafrecht analysiert (Drumbl 2013). Greisers Prozess zeigt auf, dass WSK-Rechtsaspekte durchaus bereits in den frühen Prozessen des modernen Völkerstrafrechts eine z.T. beträchtliche Rolle spielten.

Auch weitere Verfahren nach dem Zweiten Weltkrieg behandelten Sachverhalte, die man heutzutage als WSK-Rechtsverletzungen qualifizieren würde: Im bekannten Nürnberger Verfahren gegen Karl Brandt und weitere Nazi-Ärzte ging es unter anderem um unfreiwillige, grauenhafte, mitunter tödliche Menschenversuche, die Kriegsverbrechen und Verbrechen gegen die Menschlichkeit darstellen und gleichzeitig zweifelsohne das Recht auf Gesundheit verletzten (Brandt et al. [sog. Ärzteprozess], II Reports of Trials of War Criminals Before the Nuremberg Military Tribunals Under Control Council Law No. 10 (TWC): 171).

Im ebenfalls bekannten »Juristenprozess « beschäftigte sich das Gericht in Nürnberg unter anderem auch mit Gesetzgebung, die Juden pauschal vom öffentlichen Dienst und von Bildungseinrichtungen ausschloss. Ein Staat, welcher solche Gesetzgebung erlässt, verletzt das völkerrechtlich verankerte Recht auf Arbeit sowie das Recht auf Bildung (Altstoetter et al. [sog. Juristenprozess], III TWC: 954).

Im fünften Nürnberger Nachfolgeprozess wurden Friedrich Flick und andere insbesondere für die Ausbeutung von SklavenarbeiterInnen verurteilt. In diesem Urteil hielt das Gericht außerdem fest, dass der Tatbestand der Verfolgung erfüllt sein könne, wenn sich Eigentumszerstörungen gegen die Behausungen und Nahrungsvorräte der Bevölkerung richteten (United States of America v. Flick et al., VI TWC1187, NMT: 1214-1215). Wenn ein Staat für solche Zerstörungen verantwortlich ist, begeht der einzelne Täter ein Verbrechen gegen die Menschlichkeit, während der Staat gleichzeitig das Recht auf angemessenen Wohnraum und das Recht auf Nahrung verletzt.

Die Schriften von Raphael Lemkin, dem geistigen Vater der Genozid-Konvention, weisen ebenfalls mit Nachdruck auf die Relevanz soziökonomischer und kultureller Dimensionen hin. Lemkin beschreibt in seinem Buch Axis Rule in Europe (Lemkin 1944: insb. 87-89), wie die Erschwerung von sozioökonomischen Lebensbedingungen oder die Einschränkung der kulturellen Rechte der betroffenen Bevölkerung sehr wohl geeignet sein können, schwerste Verbrechen durchzuführen.

Die Beispiele, welche eine Überschneidung zwischen völkerstrafrechtlichen Tatbeständen und WSK-Rechtsverletzungen illustrieren, sind nicht auf den Zweiten Weltkrieg beschränkt. Aus Platzgründen muss es genügen, bloß zwei weitere, aktuellere Beispiele zu nennen. Erstens hat das Tribunal für das ehemalige Jugoslawien z.B. Angeklagte für Kriegsverbrechen und Verbrechen gegen die Menschlichkeit verurteilt, weil sie unter anderem in diskriminierender Weise Personen aus Betrieben entlassen haben, innen z.T. die Strom- und Wasserzufuhr abstellten und sie und inre Familien so dazu zwangen, ein bestimmtes Gebiet zu verlassen (Prosecutor v. Krajišnik, IT00-39-T, 27. September 2006: 271, Ziff. 729). Der unterlassene Schutz vor solchen Praktiken kann als Verletzung des Rechts auf Arbeit und des Rechts auf einen angemessenen Lebensstandard qualifiziert werden.

Im zweiten hier erwähnten Fallbeispiel verstand die Strafkammer des IStGH im Prozess gegen Germain Katanga, dass der Diebstahl von gewöhnlichen (und aus unserer Sicht vielleicht wenig wertvollen) Haushaltsgegenständen und rudimentärem Baumaterial in einem Konfliktgebiet wie Ituri in der Demokratischen Republik Kongo für die konkreten sozioökonomischen Lebensbedingungen der Betroffenen durchaus so schwerwiegend sein kann, dass die Tatbestandselemente der Plünderung erfüllt sein können (Procureur c. Katanga, Jugement rendu en application de l'article 74 du Statut, 7 . März 2014, Chambre de première instance II, $N^{\circ}$ ICC01/04-01/07: 382-384, Ziff. 953-957). 
Zur Überschneidung zwischen völkerstrafrechtlichen Verbrechen und WSK-Rechten lässt sich zusammenfassend festhalten, dass VölkerstrafrechtlerInnen sich legitimerweise mit vielen Aspekten von WSK-Rechten befassen können. Die oben genannten Beispiele zeigen auf, dass WSK-Rechtsmissbräuche seit den Anfängen des modernen Völkerstrafrechts Teil dieses Rechtsgebietes sind. Da die von Fatou Bensouda erwähnten Probleme (Umweltzerstörung, illegale Ressourcenausbeutung und Landnahme) nicht selten Hand in Hand mit WSK-Rechtsverletzungen auftreten, kann der Chefanklägerin somit beigepflichtet werden, dass sich der Strafgerichtshof in Den Haag diesen Sachverhalten zuwenden darf, ohne den rechtlichen Rahmen des Römischen Statuts zu überschreiten.

Ob bzw. inwiefern dies auch eine gute Idee wäre, ist Gegenstand des letzten Kapitels.

\section{Fazit}

Umweltzerstörung oder Landraub sind im Römischen Statut keine eigenständigen Straftatbestände. Daran ändert das Grundsatzpapier der Chefanklägerin selbstverständlich nichts. Sofern man die Forschungsergebnisse zur bisher kaum beachteten Überschneidung zwischen WSK-Rechten und völkerstrafrechtlichen Tatbeständen allerdings für plausibel hält, kann sich der IStGH im Rahmen seiner Zuständigkeit tatsächlich wesentlich stärker um die Ermittlung von Verbrechen gegen die Menschlichkeit kümmern, bei denen es unter anderem um Landraub, Umweltzerstörung oder illegale Ressourcenausbeutung und die Zerstörung oder Erschwerung von Lebensgrundlagen einer Bevölkerung geht.

Nur weil es eine Überschneidung zwischen WSK-Rechtsverletzungen und dem Völkerstrafrecht gibt, heißt das jedoch selbstverständlich noch lange nicht, dass Strafgerichtshöfe in Den Haag und in den nationalen Rechtssystemen, Untersuchungs- und Wahrheitskommissionen oder NGOBerichte mit völkerstrafrechtlichen Analysen sich nun in unkritischer Weise auf WSKRechtsverletzungen konzentrieren sollen. Aus der rechtlichen Analyse lässt sich »nur « schließen, dass ein juristisches Instrumentarium besteht, WSK-Rechtsverletzungen im Rahmen völkerstrafrechtlicher Überlegungen zu thematisieren. Die Hauptforderung, die sich aus dem Nachweis der Überschneidung zwischen WSK-Rechten und völkerstrafrechtlichen Tatbeständen ergibt, ist demnach die Forderung, dass mutmaßliche Straftaten in sozioökonomischen oder kulturellen Lebensbereichen ernst genommen werden müssen - und zwar aus den gleichen Gründen, aus denen wir andere völkerstrafrechtliche Verbrechen ernst nehmen. Für Juristen und Juristinnen zeigt meine Arbeit, dass es nicht akzeptabel ist, von Missbräuchen in WSKRechtsbereichen betroffenen Personen zu sagen, dass das Völkerrecht einen nahezu exklusiven Fokus auf wenige zivile und bürgerliche Rechte erzwinge und man deshalb leider nichts tun könne, damit sie ihr Schicksal völkerrechtlich zur Sprache bringen könnten. Für Nicht-Juristen und Juristinnen spielt es ebenfalls eine wesentliche Rolle, ob sie z.B. den Hunger in Kambodscha während dem Khmer-Regime als Resultat eines mutmaßlichen Verbrechens beschreiben, oder ob der Hunger als Kontextinformation dargestellt wird. Werden WSK-Rechtsmissbräuche nämlich unreflektiert als Hintergrundinformationen dargestellt, ist dies ein Signal gegenüber der betroffenen Bevölkerung, dass diese Missbräuche als Resultat unglücklicher Umstände zu ertragen seien. Eine solche Darstellung ist mitunter Wasser auf die Mühlen derjenigen, die WSK-Rechte als bloße politische Schönwetterambitionen darstellen und innen den rechtlichen Gehalt absprechen wollen.

Zudem sendet das Strategiepapier eine wichtige Nachricht an die nationalen Strafjustizen. Die nationalen Gerichte in allen Staaten, welche das Statut des IStGH ratifiziert haben, bleiben zentrale Akteure im Völkerstrafrecht. Solange die nationale Strafjustiz Fälle selber plausibel bearbeitet, erlangt der IStGH keine Zuständigkeit (Römisches Statut, Art. 17). Die Ankündigung der Chefanklägerin betreffend Umweltzerstörung, Ressourcenausbeutung und Landnahme kann somit 
auch als Aufruf verstanden werden, dass sich auch die Völkerstrafrechtlerlnnen in den nationalen Rechtssystemen und in der juristischen Ausbildung vermehrt mit solchen Themen beschäftigen sollten. Bei aller positiven Würdigung des Strategiepapiers dürfen jedoch die Grenzen des Völkerstrafrechts als Instrument gegen Umweltzerstörung, illegale Ressourcenausbeutung oder Landraub und als Werkzeug des sozialen und politischen Wandels ganz allgemein nicht unterschätzt werden. So wäre es unrealistisch, zu erwarten, dass sich kurzfristig am IStGH viel verändern wird. Zu groß sind die gravierenden Herausforderungen, die sich dem IStGH stellen. Nicht alle dieser Herausforderungen können zudem vom Gerichtshof kontrolliert werden, sondern sind auf strukturelle Eigenheiten der Internationalen Beziehungen zurückzuführen (so insbesondere das Problem der fehlenden Möglichkeiten, Haftbefehle durchzusetzen und unwillige Staaten zur Kooperation zu bewegen). Der IStGH und die multilateralen Bemühungen für eine globale Justiz und das Völkerrecht überhaupt befinden sich gegenwärtig in einer Krise, deren Ausgang im Kontext der bedenklichen internationalen Entwicklungen in Europa und den USA noch nicht abschätzbar sind. Erfolgreiche Versuche, das Budget des IStGHs empfindlich zu beschränken (Brown 2016) und die Enttäuschungen über die ersten fünfzehn Jahre des Gerichtshofes, sowie die bereits erfolgten Austritte aus dem System könnten zu einem zusätzlichen Rückgang der Unterstützung für die Institutionen des Völkerstrafrechts führen. Gegen diese politische Großwetterlage wird auch ein nichtafrikanischer Fall zu Umweltzerstörung oder Ressourcenausbeutung sehr wenig ausrichten können.

Zudem ist nicht von der Hand zu weisen, dass mit einer angekündigten Öffnung in Richtung soziökonomischer Sachverhalte weiterhin Situationen aus dem Globalen Süden im Zentrum der Aufmerksamkeit des Gerichtshofes sein dürften und sich mit der Ankündigung Fatou Bensoudas nicht alle Schwierigkeiten bewältigen lassen. So muss man davon ausgehen, dass die positiven Reaktionen auf das Strategiepapier im Tonfall tendenziell übertrieben optimistisch ausgefallen sind. Insbesondere die Organisationen hinter der erwähnten Dokumentation der mutmaßlichen Verbrechen in Kambodscha verfolgen (legitimerweise) eigennützige Interessen, indem sie in ihren Medienmitteilungen euphorisch den Eindruck zu vermitteln versuchen, dass der IStGH kurz davorsteht, eine Untersuchung zu Kambodscha zu eröffnen. Die Darstellung der Ankündigung Bensoudas als bahnbrechendes Zeichen, »dass sich das Zeitalter der Straflosigkeit dem Ende nähert « (Gillian Caldwell gegenüber der Agence France Press 2016) scheint deshalb überzeichnet und trägt den Grenzen der Möglichkeiten des Völkerstrafrechts im Allgemeinen und den Grenzen des IStGHs im Besonderen zu wenig Rechnung.

Immerhin leistet das Strategiepapier aber einen längst fälligen Beitrag für eine ehrlichere Auseinandersetzung zur Frage, wie ein internationaler Gerichtshof wie der IStGH Fälle auswählen sollte. Denn je mehr Völkerstrafrechtlerlnnen und andere davon ausgehen, dass ein rechtliches Instrumentarium grundsätzlich vorhanden ist, sich mit der Zerstörung oder Erschwerung von Lebensgrundlagen, Umweltschäden oder Landnahme und damit einhergehenden WSKRechtsproblemen zu befassen, je eher können die Möglichkeiten des bestehenden Völkerstrafrechts dort ausgeschöpft werden, wo es sinnvoll erscheint, ohne dabei der überhöhten Erwartung zu verfallen, dass ein Bezug auf das Völkerstrafrecht die Gesamtheit aller Schwierigkeiten je wird bewältigen können.

Literatur

Agence France Press 2016: ICC Prosecutors to Step up Focus on Ecological Crimes, in:

http://www.japantimes.co.jp/news/2016/09/16/world/icc-prosecutors-step-focus-ecological-crimes/\#, 16.2.2017. 
Andreu-Guzman, Federico 2012: Criminal Justice and Forced Displacement: International and National Perspectives, in: Duthie, Roger (Hrsg.): Transitional Justice and Displacement, New York, 233-253.

Arbour, Louise 2007: Economic and Social Justice for Societies in Transition, in: New York University Journal of International Law and Politics, 40, 1-28.

ASP 2016: Resolution ICC-ASP/15/Res.5, in: ICC-ASP/15/20/Vol. I, 24.11.16.

Brown, Matthew 2016: African Withdrawals Mask the Real Issue at the Assembly of States Parties 15), in: http://opiniojuris.org/2016/12/08/african-withdrawals-mask-the-real-issue-at-the-assembly-of-states-parties-15/; 16.2.2017.

Cahill-Ripley, Amanda 2014: Foregrounding Economic and Social Rights in Transitional Justice. Realising Justice for Violations of Economic, Social and Cultural Rights, Netherlands Quarterly of Human Rights, 32, 183-213.

Cruvellier, Thierry 2016: For I.C.C., a New Shift Out of Africa, in: New York Times, 6.11.16, A1. DeGuzman, Margaret 2012: Choosing to Prosecute. Expressive Selection at the International Criminal Court, in: Michigan Journal of International Law, 33, 265-320.

Drumbl, Mark 2013: „Germans Are the Lords and Poles Are the Servants «. The Trial of Arthur Greiser in Poland, 1946, in: Heller, Kevin/Simpson, Gerry (Hrsg.): The Hidden Histories of War Crimes Trials. Oxford, 411-429.

Epstein, Catherine 2010: Model Nazi: Arthur Greiser and the Occupation of Western Poland, Oxford.

Global Diligence LLP 2014: ICC Cambodian Case Study, in: http://www.globaldiligence.com/about-us/icccambodian-case-study/; 16.2.2017.

Haldemann, Frank/Kouassi, Rachelle 2014: Transitional Justice without Economic, Social and Cultural Rights?, in: Riedel/Eibe, Golay/Christophe/Mahon, Claire/Giacca, Gilles (Hrsg.): Economic, Social, and Cultural Rights: Contemporary Issues and Challenges, Oxford, 498-516. Hayner, Priscilla 2011: Unspeakable Truths: Transitional Justice and the Challenge of Truth Commissions, 2. Aufl., New York.

Herik, Larissa van den 2014: Economic, Social and Cultural Rights - International Criminal Law's Blind Spot?, in: Riedel/Eibe, Golay/Christophe, Mahon/Claire/Giacca, Gilles (Hrsg.): Economic, Social, and Cultural Rights: Contemporary Issues and Challenges, Oxford, 343-366. 
ICC Observers 2009: Interview with William Schabas, in: http://iccobservers.wordpress.com/2009/03/26/iccobservers-exclusive-interview-william-schabas-professor-of-human-rights-law-and-director-of-the-irish-centre-for-human-rights-at-the-nationaluniversity-of-ireland-galway/; 16.2.2017.

Irish Centre for Human Rights 2017: International Criminal Court Summer School 2017, in http://www.conference.ie/Conferences/index.asp?Conference=497; 16.2.17.

Kälin, Walter/Künzli, Jörg 2013: Universeller Menschenrechtsschutz, 3. Aufl., Basel.

Karnavas, Michael G. 2016: Will Launching Investigations into Non-African Situations Stem the Exodus of African States from the ICC?, in: http://michaelgkarnavas.net/blog/2016/11/07/investigating-non-africansituations/; 16.2.2017.

Kersten, Mark 2016: A Turn to the »Symbolic« at the International Criminal Court, in: https://justiceinconflict.org/2016/10/05/a-turn-to-the-symbolic-at-the-international-criminal-court/; 16.2.2017.

Kring, Franziska 2016: Environmental Destruction as Crime Against Humanity? Climate Change and Its Implications on International Criminal Law, BOFAX Nr. 489E, Institut für Friedenssicherungsrecht und Humanitäres Völkerrecht der Ruhr-Universität Bochum, 29.9.16. Labuda, Patryk I. 2016, Africa and the ICC: Shattered Taboos, and the Status Quo, in: http://www.ejiltalk.org/africa-and-the-icc-shattered-taboos-and-the-status-quo/, 16.2.2017.

Lemkin, Raphael 1944: Axis Rule in Occupied Europe, Washington.

Meijer, Tim/Glasius, Marlies 2016: Trials as Messages of Justice: What Should Be Expected of International Criminal Courts?, 30 Ethics \& International Affairs, 429-447.

Miller, Zinaida 2008: Effects of Invisibility. In Search of the »Economic«, in: Transitional Justice, International Journal of Transitional Justice, 2, 266-291.

Office of the United Nations High Commissioner for Human Rights 2014: Transitional Justice and Economic, Social and Cultural Rights, New York/Geneva.

Office of the Prosecutor of the International Criminal Court 2016a: Policy Paper on Case Selection and Prioritisation, The Hague.

Office of the Prosecutor of the International Criminal Court 2016b: Report on Preliminary Examination Activities, The Hague.

Okafor, Obiora Chinedu/Ngwaba, Uchechukwu 2014: The International Criminal Court as a "Transitional Justice« Mechanism in Africa. Some Critical Reflections, International Journal of Transitional Justice, 9, 90-108. 
Seachange, the Blog of Sea Shepherd Legal 2016: International Criminal Court Places New Emphasis on Addressing Destruction of the Environment, in: https://seachangessl.org/2016/09/16/internationalcriminal-court-places-new-emphasis-on-addressing-destruction-of-the-environment/; 16.2.2017.

Schmid, Evelyne 2015: Taking Economic, Social and Cultural Rights Seriously in International Criminal Law, Cambridge.

Schmid, Evelyne/Nolan, Aoife 2014: »Do No Harm «? Exploring the Scope of Economic and Social Rights in Transitional Justice, International Journal of Transitional Justice, 8, 362-382.

Schwöbel-Patel, Christine 2016: The Re-Branding of the International Criminal Court (and Why African States Are Not Falling for It), in: http://opiniojuris.org/2016/10/28/the-re-branding-of-the-international-criminal-courtand-why-african-states-are-not-falling-for-it/; 16.2.2017.

Sharp, Dustin 2012: Addressing Economic Violence in Times of Transition, Fordham International Law Journal, 35, 780-814.

Sharp, Dustin (Hrsg.) 2013: Justice and Economic Violence in Transition, New York.

Smith, Tara 2016: Why the International Criminal Court Is Right to Focus on the Environment, in: https://theconversation.com/why-the-international-criminal-court-is-right-to-focus-on-the-environment-65920, 16.2.2017.

Taylor, Adam 2016: Is Environmental Destruction a Crime against Humanity? The ICC May Be About to Find Out, in: Washington Post, 16. September 2016.

UN Secretary-General, 2010: Guidance Note of the Secretary-General on the United Nations Approach to Transitional Justice, Genf/New York.

Vereinte Nationen 1998, Römisches Statut des Internationalen Strafgerichtshofs, in: A/CONF.183/9, 17.7.1998, amtliche Übersetzung des Auswärtigen Amtes der Bundesrepublik Deutschland.

Werle, Gerhard 2016: Völkerstrafrecht, Tübingen. 